Research, Society and Development, v. 9, n. 11, e53091110200, 2020

(CC BY 4.0) | ISSN 2525-3409 | DOI: http://dx.doi.org/10.33448/rsd-v9i11.10200

\title{
Uso de Wearable em pacientes com lombalgia
}

Use of Wearable in patients with low back pain

Uso de Wearable en pacientes con lombalgia

Recebido: 13/11/2020 | Revisado: 16/11/2020 | Aceito: 20/11/2020 | Publicado: 25/11/2020

\section{Rafael Victor Ferreira do Bonfim}

ORCID: https://orcid.org/0000-0002-1960-1282

Universidade Brasil, Brasil

E-mail: ft.rafaelvictor@hotmail.com

Thayanne Kelly de Sousa Cabral

ORCID: https://orcid.org/0000-0003-1978-9681

Universidade Estácio de Sá, Brasil

E-mail: fisiothayannecabral@gmail.com

Rayane Portela de Lima

ORCID: https://orcid.org/0000-0003-3859-8119

Universidade Estácio de Sá, Brasil

E-mail: ray.aneportela@ hotmail.com

Bruno da Silva Gomes

ORCID: https://orcid.org/0000-0002-4527-3956

Universidade Estácio de Sá, Brasil

E-mail: bsgomes100@gmail.com

Gabriel Mauriz de Moura Rocha

ORCID: https://orcid.org/0000-0003-1454-0414

Universidade Estácio de Sá, Brasil

E-mail: mauriz45@hotmail.com

Lívia Assis

ORCID: https://orcid.org/0000-0002-8343-3375

Universidade Brasil, Brasil

E-mail: livia.assis@universidadebrasil.edu.br

Vilson Rosa Almeida

ORCID: https://orcid.org/0000-0001-9077-2941

Universidade Brasil, Brasil

E-mail: vilson.almeida@universidadebrasil.edu.br 


\title{
Resumo
}

A presente pesquisa tem por objetivo avaliar o efeito do tratamento para lombalgia utilizando sensores de movimento, por meio da análise do nível de dor, verificação de Amplitude De Movimento (ADM) da coluna, e grau de incapacidade física no pré e pós tratamento. Os pacientes foram submetidos a avaliação pré e pós tratamento por meio da Escala Verbal Numérica para mensurar nível de dor, estabilometria para avaliação da estabilidade, questionário de Roland-Morris de Incapacidade para nível de funcionalidade e goniometria para mensuração de Amplitude de Movimento. No tratamento foram realizadas uma sessão por dia, durante 10 dias consecutivos, com duração de 30 minutos cada sessão. Os resultados demonstram que houve diminuição do nível de dor nos pacientes $(P=0,0008)$. Além disso, nível de funcionalidade também diminuiu significativamente $(P=0,001)$ e discreto aumento dos valores goniométricos. Por meio dos resultados obtidos, conclui-se que o uso de Wearable em pacientes com lombalgia se mostra eficaz no tratamento da enfermidade, possibilitando alivio da dor, aumento da amplitude de movimento e melhora da capacidade funcional dos pacientes.

Palavras-chave: Dor lombar; Coluna vertebral; Fisioterapia.

\begin{abstract}
The present research aims to evaluate the effect of treatment for low back pain using motion sensors, by analyzing the level of pain, verifying the range of motion (ROM) of the spine, and the degree of physical disability in the pre and post treatment. Patients underwent pre- and post-treatment assessment using the Numerical Verbal Scale to measure pain level, stabilometry to assess stability, Roland-Morris Disability questionnaire for level of functionality and goniometry to measure Range of Motion. In treatment, one session per day was carried out for 10 consecutive days, each session lasting 30 minutes. The results demonstrate that there was a decrease in the level of pain in the patients $(\mathrm{P}=0.0008)$. In addition, the level of functionality also decreased significantly $(P=0.001)$ and a slight increase in goniometric values. Through the results obtained, it is concluded that the use of Wearable in patients with low back pain proves to be effective in the treatment of the disease, allowing for pain relief, increased range of motion and improved functional capacity of patients.
\end{abstract}

Keywords: Low back pain; Spine; Physiotherapy. 


\section{Resumen}

La presente investigación ten como obxectivo avaliar o efecto do tratamento da dor lumbar mediante sensores de movemento, analizando o nivel de dor, verificando o rango de movemento (ROM) da columna vertebral e o grao de discapacidade física no tratamento previo e posterior. Os pacientes sometéronse a avaliación previa e posterior ao tratamento mediante a escala verbal numérica para medir o nivel de dor, a estabilometría para avaliar a estabilidade, o cuestionario de discapacidade Roland-Morris para o nivel de funcionalidade e a goniometría para medir o rango de movemento. No tratamento, levouse a cabo unha sesión ao día durante 10 días consecutivos, cada sesión durou 30 minutos. Os resultados demostran que houbo unha diminución do nivel de dor nos pacientes $(\mathrm{P}=0,0008)$. Ademais, o nivel de funcionalidade tamén diminuíu significativamente $(\mathrm{P}=0,001)$ e un lixeiro aumento dos valores goniométricos. A través dos resultados obtidos, conclúese que o uso de Wearable en pacientes con dor lumbar demostra ser eficaz no tratamento da enfermidade, permitindo aliviar a dor, aumentar o rango de movemento e mellorar a capacidade funcional dos pacientes.

Palabras clave: Dor lumbar; Espiña dorsal; Fisioterapia.

\section{Introdução}

A dor lombar (LBP) é um importante problema de saúde, sendo uma das principais causas de incapacidade no mundo. Devido a sua prevalência, de aproximadamente $70 \%$ da população mundial, é considerada um problema de saúde pública em todo o planeta, gerando impactos socioeconômicos consideráveis (Akindele-Agbeja et al., 2017; Sowden et al., 2018; Stollenwerk et al., 2019).

Os distúrbios musculoesqueléticos são as principais causas da dor lombar. Os músculos paravertebrais, particularmente os multífidos, têm um importante papel biomecânico na estabilização da postura e limitação do movimento intervertebral excessivo. (Bláfoss et al., 2019; Agha et al., 2020). As fibras profundas desses músculos são as primeiras a serem ativadas durante o movimento de um membro qualquer no corpo, além de controlarem o movimento vertebral e protegerem as estruturas articulares, discos, ligamentos das tensões e cargas excessivas. Outro músculo importantíssimo na estabilização postural é o transverso do abdômen, que por ter inserção na fáscia toracolombar, é o maior responsável pelo aumento da pressão intra-abdominal e junto as fibras profundas do multífido, é o 
primeiro a ser ativado no corpo durante atividades dos membros inferiores (Santos et al., 2011).

Em virtude de a dor lombar ser responsável por grandes índices de incapacidade e faltas ao trabalho, gerando alto custo para a sociedade e para os sistemas de saúde nos países desenvolvidos, diversas pesquisas têm sido conduzidas nos últimos anos buscando compreender mais sobre a dor lombar e as formas de administrá-la. As dores da coluna (cervical, torácica, lombar e pélvica) são a segunda condição de saúde mais prevalente do Brasil (13,5\%), entre as patologias crônicas identificadas por algum médico ou profissional de saúde, superadas apenas pelos casos de hipertensão arterial (14\%) (Nascimento \& Costa, 2015).

Dentre as formas mais recentes de tratamento para a dor lombar, destaca-se o uso da realidade virtual (RV), que fornece um ambiente imersivo 3D multissensorial e estimula os sentidos visuais, auditivos e de propriocepção, gerando assim a experiência perceptual de envolvimento com o ambiente virtual que está vendo, além de ser uma oportunidade de expor com segurança e de forma gradual indivíduos com dor crônica a movimentos que normalmente evitariam no mundo real (Fowler et al 2019; Patiño; Khoshnam; Menon, 2020).

Trabalhos recentes evidenciaram a eficácia da RV para a reabilitação física de condições dolorosas da coluna e para reabilitação ortopédica em termos de redução da dor (Ahern et al 2020). O uso de Wearable, dispositivos vestíveis, podem ser promissores no ambiente clínico para prevenir, monitorar e tratar problemas da coluna vertebral, bem como outros problemas musculoesqueléticos (Chakravorty, 2019).

Diante do exposto, sugere-se que a diminuição e a prevenção de lombalgias poderiam diminuir os gastos governamentais e pessoais, já que grande parte da população teve ou terá essa condição. Neste contexto, o objetivo da presente pesquisa foi avaliar o efeito do tratamento para lombalgia ultilizando sensores de movimento por meio da análise do nível de dor, verificação da Amplitude De Movimento (ADM) da coluna e do grau de incapacidade física desses pacientes, pré e pós-tratamento.

\section{Metodologia}

O tipo de estudo em questão se refere a um estudo de campo experimental, com abordagem quantitativa. 
A coleta de dados desta pesquisa foi efetuada e desenvolvida em uma Clínica de Fisioterapia privada, na cidade de Teresina-PI. O local foi escolhido devido ser uma referência em fisioterapia na cidade capital.

O tratamento foi realizado com 16 participantes. Foram incluídos na pesquisa pacientes que apresentam lombalgia, com quadro crônico, de ambos os gêneros, entre 18 e 45 anos. Foram excluídos da pesquisa pacientes que apresentam distúrbios vestibulares ou neurológicos, cujos sintomas afetem consideravelmente o equilíbrio, impossibilitando a utilização do sensor no mesmo. A coleta de dados foi realizada através de anamnese, onde os pontos abordados foram: dor, amplitude de movimento e nível de funcionalidade.

Para avaliar a dor utilizou-se uma escala verbal numérica, de 0 a 10, representando dor fraca se a intensidade for menor ou igual a 3, dor moderada se de 4 a 6 , dor intensa se de 7 a 9 e dor insuportável se igual a 10 (Drummond, 2000). Para avaliar amplitude de movimento antes e depois da terapia utilizou-se goniômetro, fazendo as mensurações após movimentos da coluna de extensão, flexão, inclinação lateral e rotação direita e esquerda. Para avaliar nível de funcionalidade utilizou-se o Questionário Roland-Morris de Incapacidade (QIRM).

QIRM foi inicialmente desenvolvido para medir incapacidade física em forma de auto relato para pacientes com dor na coluna. Tem 24 itens com pontuações de 0 ou 1 e o total varia de 0 (sugerindo nenhuma incapacidade) a 24 (incapacidade grave). O QIRM é uma medida simples, geralmente leva 5 minutos e é fácil de calcular e analisar. Muitos estudos descrevem o QIRM com boa validade, confiabilidade e responsividade para a medida de incapacidade física em pacientes com dor crônica, quando comparado a outras (Júnior et al., 2010). Para a realização do tratamento utilizou-se o aparelho de gameterapia Valedo®, onde foram colocados os sensores na área lombar e na área de externo como ilustrado na Figura 1.

Figura 1. Sensor Valedo®.

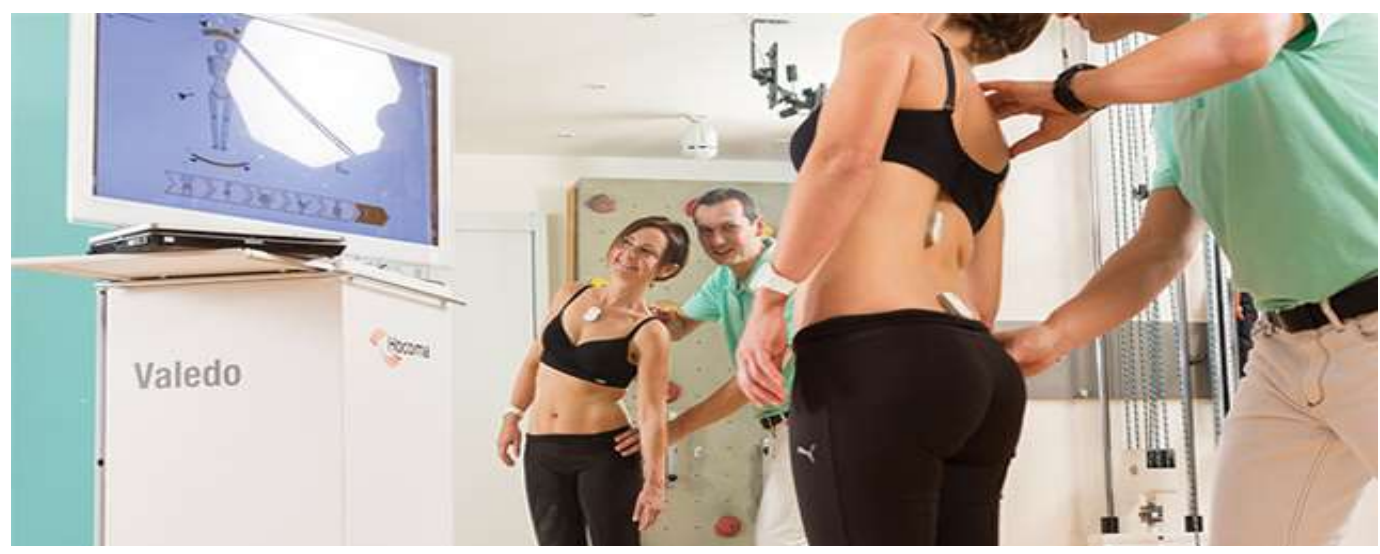

Fonte: https://knowledge.hocoma.com/training-material/valedomotion/instruction-video.html 
Research, Society and Development, v. 9, n. 11, e53091110200, 2020

(CC BY 4.0) | ISSN 2525-3409 | DOI: http://dx.doi.org/10.33448/rsd-v9i11.10200

O jogo se dá por meio de fases onde o paciente precisa chegar a determinada pontuação para avançar para o próximo nível. Em cada fase o paciente realizou movimentos com o corpo onde o enfoque deu-se na musculatura posterior lombar e abdominal, principalmente em musculatura de multífidos e transverso do abdômen, responsáveis pela estabilidade lombar. Existem 6 fases no jogo ilustradas na Figura 2.

Figura 2. Fases do jogo.

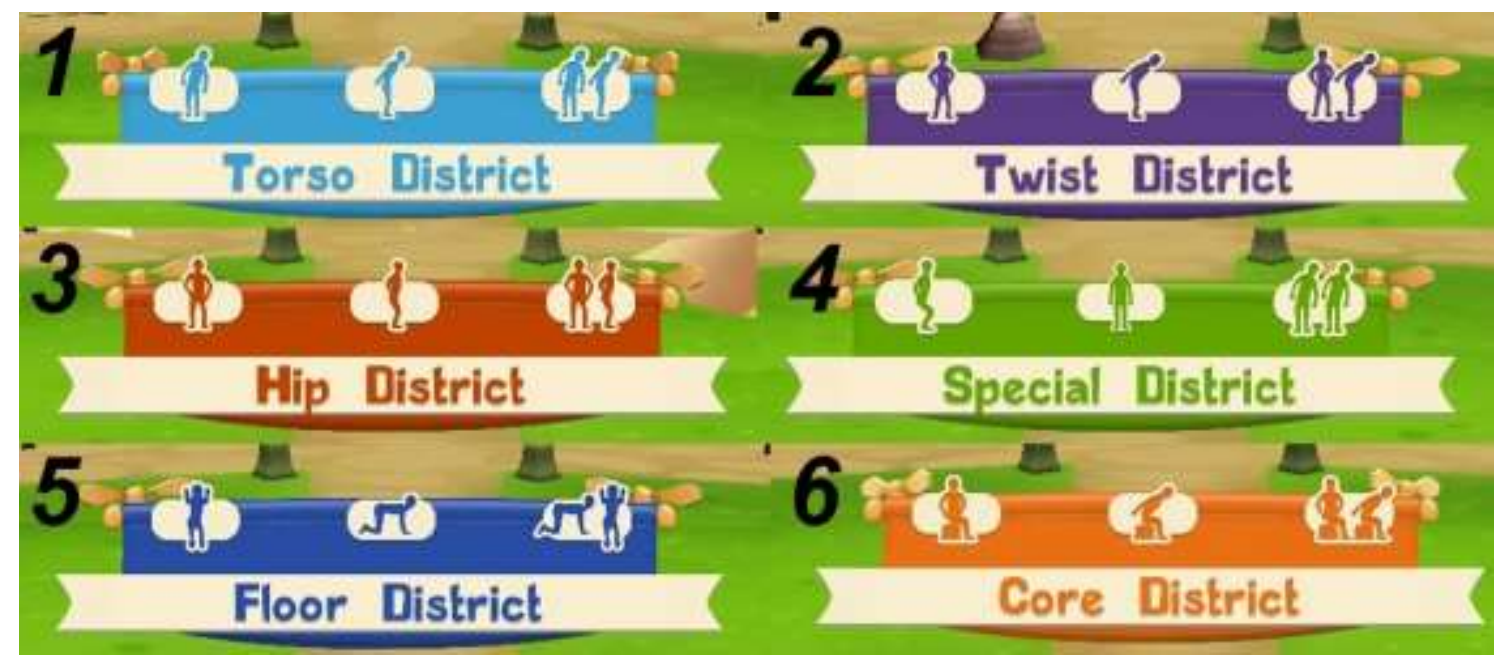

Fonte: Valedo, (2018).

Durante as fases existe um nível de dificuldade diferente, sendo gradual da primeira à última fase. Na primeira fase o participante fez movimentos de flexão, extensão e lateral direita e esquerda da coluna em posição ortostática, a segunda fase movimentos de flexão e extensão com maior amplitude em posição ortostática, na terceira fase movimentos de antiversão, retroversão e inclinação lateral direita e esquerda em posição ortostática, na quarta fase movimentos de inclinação lateral e agachamento. Na quinta fase movimentos de antiversão e retroversão em posição de gato e inclinação lateral da pelve em posição ortostática, na sexta e última fase movimentos de flexão em posição sentada. Cada sessão durou 30 minutos e cada paciente fez uma sessão por dia, durante 10 dias consecutivos.

Os dados foram organizados em planilhas do programa Microsoft Office Excel 2016 e distribuídos conforme os objetivos estabelecidos. Posteriormente os mesmos foram transferidos para o programa estatístico SpSS 20.0 onde foram submetidos aos testes estatísticos T de Studant, após a realização do teste de normalidade D’Agostine e Pearson, para as variáveis aleatórias contínuas, e teste de Pearson Qui-quadrado, para as variáveis 
aleatórias discretas. Ambos os testes com Intervalo de Confiança (IC) de 95\% e significância em $\mathrm{P}<0,05$. Os mesmos foram apresentados em tabelas e gráficos.

Todas as condutas realizadas pelo pesquisador obedeceram às diretrizes da Resolução 466/12 do Ministério da Saúde, com parecer N4005957. Objetivou-se, com isso, a proteção dos sujeitos participantes, preservando os seus direitos, segurança, bem-estar, integridade e sigilo. A pesquisa só foi realizada após os participantes assinarem o Termo de Consentimento Livre e Esclarecido (TCLE) (Pereira et al., 2018).

\section{Resultados}

A dor de cada paciente foi avaliada por meio da Escala Visual Analógica - EVA, antes e depois da intervenção com os participantes da pesquisa. Após o tratamento verificouse significativa diminuição do nível de dor dos participantes, com o valor de $\mathrm{P}$ correspondente a 0,0008 (Gráfico 1).

Gráfico 1. Avaliação da dor pré e pós tratamento pela Escala Visual Analógica - EVA.

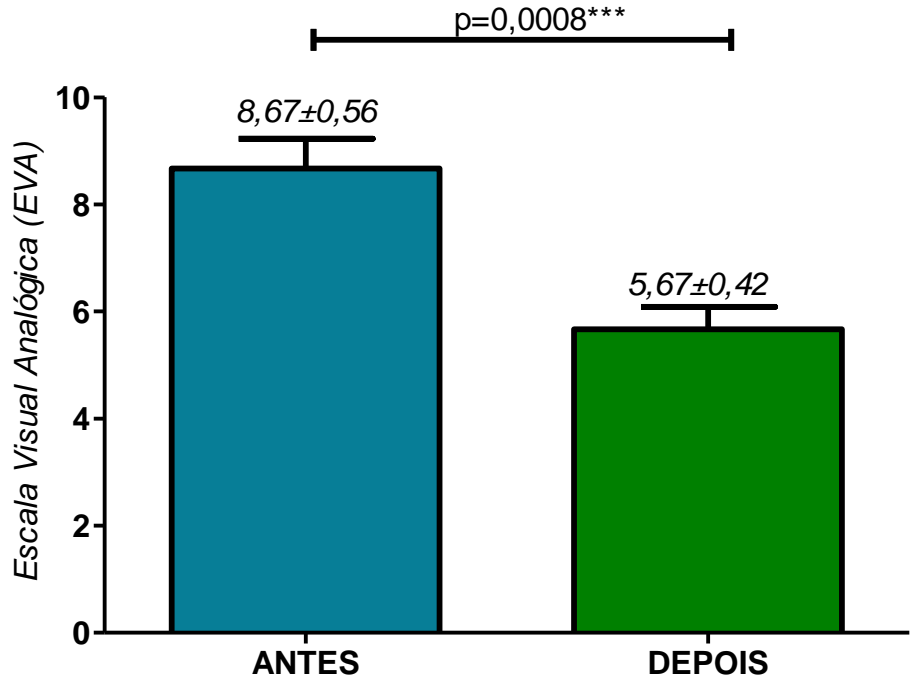

Fonte: Autores.

A avaliação da amplitude mostrou um discreto aumento da amplitude em todos os movimentos, embora sem significância estatística. 
Research, Society and Development, v. 9, n. 11, e53091110200, 2020

(CC BY 4.0) | ISSN 2525-3409 | DOI: http://dx.doi.org/10.33448/rsd-v9i11.10200

Tabela 1. Avaliação goniométrica dos pacientes pré e pós-tratamento.

\begin{tabular}{|c|c|c|c|c|c|c|}
\hline \multicolumn{2}{|c|}{ VARIÁVIES } & $\mathbf{M}$ & DP & $\operatorname{Max}$ & Min & $\mathbf{P}$ \\
\hline \multicolumn{7}{|l|}{ Flexão } \\
\hline & Antes & 70,23 & 22,00 & 105,00 & 51,40 & \multirow{2}{*}{0,3503} \\
\hline & Depois & 75,25 & 21,92 & 109,10 & 55,70 & \\
\hline \multicolumn{7}{|l|}{ Extensão } \\
\hline & Antes & 41,85 & 15,54 & 66,10 & 18,30 & \multirow{2}{*}{0,4822} \\
\hline & Depois & 42,27 & 15,95 & 68,20 & 19,30 & \\
\hline \multicolumn{7}{|c|}{ Flexão Lateral Direita } \\
\hline & Antes & 28,85 & 10,68 & 46,10 & 12,80 & \multirow{2}{*}{0,292} \\
\hline & Depois & 32,15 & 9,48 & 49,00 & 21,90 & \\
\hline \multicolumn{7}{|c|}{ Flexão Lateral Direita } \\
\hline & Antes & 30,47 & 9,43 & 43,00 & 20,30 & \multirow{2}{*}{0,3011} \\
\hline & Depois & 33,45 & 9,77 & 46,00 & 18,70 & \\
\hline \multicolumn{7}{|l|}{ Rotação Direita } \\
\hline & Antes & 63,68 & 12,43 & 79,80 & 47,50 & \multirow{2}{*}{0,4214} \\
\hline & Depois & 65,32 & 15,22 & 82,20 & 40,70 & \\
\hline \multicolumn{7}{|l|}{ Rotação Esquerda } \\
\hline & Antes & 60,00 & 15,74 & 84,20 & 34,50 & 0,3199 \\
\hline & Depois & 63,95 & 12,41 & 87,50 & & \\
\hline
\end{tabular}

Fonte: Autores.

De acordo com os resultados obtidos por meio do questionário de Roland-Morris Disability Questionnaire, com pontuações variando de 0 ("sem deficiência") a 24 ("alta deficiência”). Após o tratamento notou-se significativa diminuição do nível de dor dos participantes, com o valor de $\mathrm{P}$ correspondente a 0,001 (Gráfico 2).

Gráfico 2. Índice geral do questionário de Roland-Morris de Incapacidade.

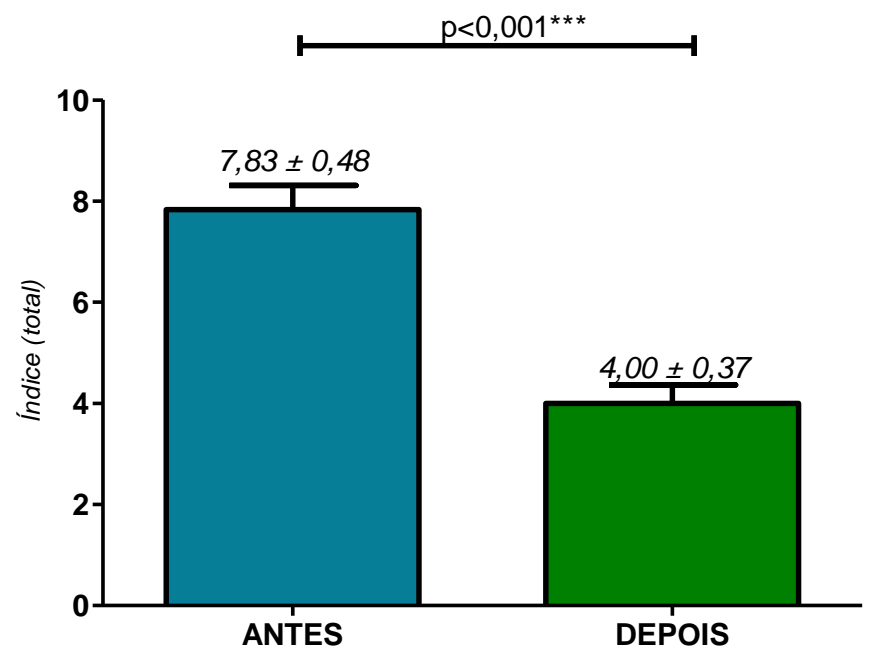

Fonte: Autores.

Medido com o Roland-Morris Disability Questionnaire, com pontuações variando de 0 ("sem deficiência") a 24 ("alta deficiência"). 


\section{Discussão}

Este estudo foi conduzido a fim de se investigar os efeitos de terapia com uso da Realidade Virtual, em pacientes com dor lombar crônica não específica investigando a possível melhoria na intensidade da dor, na incapacidade e na amplitude de movimento lombar.

A lombalgia é uma afecção bastante comum em indivíduos com fraqueza em músculos estabilizadores da coluna, e o fortalecimento destes é um dos pontos para o tratamento. Muitos autores relacionam o fato de músculos estabilizadores como multífidos e transverso do abdômen estarem ligados diretamente ao relato de dor em muitos indivíduos que possuem lombalgia inespecífica. Pacientes com dor crônica têm um viés de atenção à dor, embora as diferenças com pessoas saudáveis sejam pequenas e dependam dos métodos usados para medi-la. Essa atenção seletiva à dor e a dificuldade de se desvencilhar dela pode ser motivada por cognições e emoções relacionadas à dor, como catastrofização da dor e medo relacionado à dor. Portanto, foi levantada a hipótese de que esses fatores podem afetar a hipoalgesia induzida por distração (Matheve et al., 2020).

Nos resultados coletados no presente estudo, verificou-se que houve significativa melhora na dor lombar dos pacientes, em comparação com os valores antes e após a submissão destes ao treinamento de estabilização.

De acordo com Niederer e Mueller (2020) e Nieder et al. (2020), os exercícios de estabilização são formas de tratamento seguro e eficaz podendo reduzir o nível de deficiência devido a melhora sustentável na intensidade da dor e na incapacidade em pacientes da dor lombar crônica.

Diversos benefícios, tais como redução da dor, melhorada capacidade funcional, aumento da amplitude de movimento e melhora da força da musculatura do tronco foram encontrados nos estudos de Bhadauria e Gurudut (2017) que utilizaram exercícios com movimentos específicos da coluna vertebral capazes de ativar a musculatura estabilizadora do tronco.

Dentre as alterações biofísicas, o controle motor exerce fundamental importância na estabilização vertebral e seu treinamento por meio de movimentos da coluna se mostra promissor para o tratamento da dor lombar crônica (Shahvarpour et al., 2018).

Os músculos locais são capazes de controlar a rigidez e a relação intervertebral dos segmentos espinhais e da postura da coluna lombar. Evidências de estudos biomecânicos, morfológicos, histoquímicos, eletromiográficos e de fibras musculares têm implicado os 
músculos multífidus em 2/3 da estabilidade segmentar da coluna. A instabilidade segmentar pode causar distúrbios funcionais e tensão, bem como dor. Os músculos segmentares da coluna lombar, como o músculo multífido, podem fornecer controle segmentar e são os estabilizadores segmentares primários da coluna vertebral na região lombar. Um estudo identificou atrofia seletiva da ML após o primeiro episódio de dor nas costas; era improvável que a atrofia revertesse sem treinamento específico, e a estabilidade muscular mais baixa predispôs o indivíduo a novos episódios de lombalgia (Soundararajan \& Thankappan, 2016).

A contração dos transverso do abdômen e multífidos ocorrem normalmente de maneira antecipada aos movimentos dos membros em indivíduos assintomáticos, porém, naqueles indivíduos com dor lombar, essa contração é lenta e atrasada indicando um potencial para a redução da estabilidade da coluna e problemas com o controle motor (Macedo et al, 2009).

Há evidências de que a estrutura e função dos músculos profundos do tronco estão alteradas em indivíduos com dor lombar, por isso recentemente tem havido um maior foco nos exercícios que visam manter essa estabilidade, melhorando o controle neuromuscular, diminuindo assim a recorrência da dor (Herbert et al, 2010).

A instabilidade lombar surge então como um fator de risco para o surgimento de lombalgia, já que esses músculos têm como função principal gerar estabilidade para a coluna, em especial a lombar. Existem evidências convincentes de que a realização de um programa de exercícios com ênfase no fortalecimento da musculatura extensora do tronco restaura a função da coluna lombar e pode prevenir o surgimento da lombalgia (Maia; Gurgel \& Bezerra, 2015)

A importância dessa musculatura na estabilidade da coluna reforça a necessidade do fortalecimento abdominal, mesmo por meio de exercícios simples, como deitar e sentar, pois esta musculatura, associada à musculatura torácica, participa no suporte da coluna, e 30 a 50\% das pressões exercidas sobre os discos lombares e torácicos poderiam ser diminuídas pelo fortalecimento desses músculos. Os músculos abdominais diminuem, também, a tensão de rotação e inclinação e de cisalhamento na coluna lombar, protegendo a medula espinhal lombar (Camargo et al., 2012).

Por esse motivo, para o tratamento de lombalgia, intervenções fisioterapêuticas focadas no sistema estabilizador da coluna que envolvem exercícios para os músculos transverso do abdômen, multífidos e diafragma demonstraram ser mais eficazes que a terapia manual e orientações para casa nos desfechos dor. Exercícios específicos para os 
estabilizadores causam menor recorrência de dor lombar em relação ao uso de fármacos, comprovando a eficácia dos exercícios para a lombalgia (Gouveia \& Gouveia, 2008).

Alguns autores, como Bhadauria e Gurudut (2017), também relatam o aumento da amplitude de mobilidade lombar através de exercícios com enfoque nos músculos estabilizadores em pacientes com dor lombar. Muitos dos indivíduos que sofrem dessa enfermidade deixam de realizar movimentos em sua amplitude normal pelo fato de sentirem dor ou até medo de realizar o movimento com precipitação dessa dor, o que possivelmente explica a não alteração significativa nas amplitudes de movimento da coluna dos pacientes analisados.

A incapacidade gerada pela dor torna-se mínima, já que, nesse contexto, através do treinamento de multífidos e transverso do abdômen, consegue-se diminuir consideravelmente a lombalgia.

Exercícios de controle motor (também conhecido como exercício de estabilização) foram consideradas pela primeira vez como um tratamento para dor lombar cerca de 13 anos atrás, quando um grupo de pesquisadores da Universidade de Queensland, na Austrália, publicou o primeiro artigo sobre este tópico. Desde então, o número de estudos sobre este assunto, 14-16 como bem como sua popularidade e uso na prática clínica, aumentaram (Chou \& Huffman, 2007)

O raciocínio biológico para o exercício de controle motor é fundamentalmente baseado na ideia de que a estabilidade e o controle da coluna vertebral são alterados em pessoas com dor lombar. Estudos fisiológicos demonstraram que pacientes com dor lombar podem apresentar atraso no início da atividade dos músculos profundos do tronco (por exemplo, transverso abdominal, multífidos) quando a estabilidade da coluna é desafiada em tarefas dinâmicas. Morfologicamente, uma área transversal inferior e uma porcentagem maior de gordura intramuscular no músculo multífido20 foram encontrados em pacientes com lombar dor em comparação com controles assintomáticos. Além disso, verificou-se que pacientes com lombalgia tendem a aumentar a rigidez da coluna para compensar a falta de estabilidade dos músculos profundos, aumentando a atividade dos músculos superficiais (Costa et al., 2009).

Assim, o fortalecimento dos músculos abdominais é essencial para o tratamento de dor lombar e é uma técnica comumente empregada na reabilitação por promover estabilidade da coluna lombar. Alguns programas de exercícios que melhoram a força muscular do abdômen parecem diminuir a intensidade da dor lombar, aumentar a força, mobilidade e a resistência (França, 2009). 
Research, Society and Development, v. 9, n. 11, e53091110200, 2020

(CC BY 4.0) | ISSN 2525-3409 | DOI: http://dx.doi.org/10.33448/rsd-v9i11.10200

Encontramos uma consonância entre o presente estudo e o de diversos pesquisadores que utilizaram exercícios capazes de ativar a musculatura estabilizadora da coluna e obtiveram a melhora da dor visualizada por meio da escala analógica visual de dor (Hwangbo et al., 2015), mostrando ainda que os exercícios de estabilização segmentar se mostraram mais eficazes do que programas convencionais de exercícios na redução da incapacidade funcional em pacientes com dor lombar crônica (Alhakami et al., 2019), e ainda que o tratamento utilizando esses exercícios foram úteis não só na redução da dor lombar mas também na incapacidade funcional (Sipaviciente \& Kliziene, 2020).

\section{Considerações Finais}

Após a realização do estudo em questão pode-se concluir que o uso de wearable em pacientes com lombalgia mostrou-se eficaz no tratamento da enfermidade, possibilitando alivio da dor e melhora da capacidade funcional dos pacientes, demonstrando uma significância estatística e/ou clínica, principalmente na redução da intensidade da dor, e do nível de incapacidade. Além disso, este estudo expandiu a base de conhecimento, demonstrando a viabilidade da RV como um complemento para intervenções de dor crônica a fim de melhorar a atividade e a impressão global de recuperação e para melhorar a intensidade da dor em longo prazo onde os profissionais de saúde e pesquisadores podem trabalhar lado a lado para validar cada vez mais estudo com o uso do wearables como uma ferramenta de apoio podendo ser então incorporada ao sistema de saúde como uma nova forma de tratamento.

\section{Referências}

Agha, O., Mueller-Immergluck, A., Liu, M., Zhang, H., Theologis, A. A., Clark, A., Kim, H. T., Liu X., Feeley, B. T., \& Bailey, J. F. (2020). Intervertebral disc herniation effects on multifidus muscle composition and resident stem cell populations. JOR Spine, 3(2), 1-10. https://doi.org/10.1002/jsp2.1091

Ahern MM, Dean LV, Stoddard CC, Agrawal A, Kim K, Cook CE, Narciso Garcia A. (2020). The Effectiveness of Virtual Reality in Patients With Spinal Pain: A Systematic Review and Meta-Analysis. Pain Pract. 2020 Jul;20(6):656-675. doi: 10.1111/papr.12885. Epub 2020 May 21. PMID: 32196892. 
Akindele-Agbeja, O., Mbada, C. E., \& Egwu, M. O. (2017). Does the inclusion of spinal manipulative therapy in multimodal treatment regimens result in better outcomes in chronic low back pain? A systematic review. Proceedings of Singapore Healthcare, 26(2), 114-20. https://doi.org/10.1177\%2F2010105817698491

Alhakami, A. M., Davis, S., Qasheesh, M., Shaphe, A., \& Chahal, A. (2019). Effects of McKenzie and stabilization exercises in reducing pain intensity and functional disability in individuals with nonspecific chronic low back pain: a systematic review. J Phys Ther Sci, 31(7), 590-597. https://doi.org/10.1589/jpts.31.590

Bhadauria, E. A., Gurudut, P. (2017). Comparative effectiveness of lumbar stabilization, dynamic strengthening, and Pilates on chronic low back pain: randomized clinical trial. $J$ Exerc Rehabil, 13(4), 477-485. https://doi.org/10.12965/jer.1734972.486

Bláfoss, R., Aagaard, P., \& Andersen., LL. (2019). Physical and psychosocial work environmental risk factors of low-back pain : protocol for a 1 year prospective cohort study. BMC Muscoloskelet Disord, 20(626), 1-7. https://doi.org/10.1186/s12891-019-2996-z

Camargo, G. M., Marques, A. E. Z. S., \& Pedroni, C. R. (2012). Avaliação da Dor e da Postura em Pacientes com Lombalgia Submetidos a Dois Protocolos de Fortalecimento Abdominal. Revista Terapia Manual, 10(50), 496-501.

https://repositorio.unesp.br/handle/11449/114987

Chakravorty, A., Mobbs, R.J., Anderson, D.B. (2019). The role of wearable devices and objective gait analysis for the assessment and monitoring of patients with lumbar spinal stenosis: systematic review. BMC Musculoskelet Disord 20, 288. https://doi.org/10.1186/s12891-019-2663-4

Costa, L. O. P., Maher, C. G., Latimer, J., Hodges, P. W., Herbert, R. D., Refshauge, K. M., McAuley, J. H., \& Jennings, M. D. (2009). Motor Control Exercise for Chronic Low Back Pain: A Randomized Placebo-Controlled Trial. Phys Ter, 89(12), 1275-86.

https://doi.org/10.2522/ptj.20090218 
Chou, R., Huffman, L. H. (2007). Nonpharmacologic therapies for acute and chronic low back pain: a review of the evidence for an American Pain Society/American College of Physicians clinical practice guideline. Ann Intern Med, 147(7), 492-504. https://doi.org/10.7326/0003-4819-147-7-200710020-00007

DRUMMOND, J.P. (2000). Dor aguda: fisiopatologia, clínica e terapêutica. Atheneu.

França, F. J. F. (2009). Estabilização segmentar lombar, fortalecimento e alongamento no tratamento da lombalgia crônica: um estudo comparativo. (Dissertação de mestrado, Faculdade de Medicina da Universidade de São Paulo, São Paulo, Brasil)

Gouveia, K. M. C., Gouveia, E. C. (2008). O músculo transverso abdominal e sua função de estabilização da coluna lombar. Fisioter mov, 21(3), 45-50. http://bases.bireme.br/cgibin/wxislind.exe/iah/online/?IsisScript=iah/iah.xis\&src=google\&base=LILACS\&lang=p\&nex tAction=lnk\&exprSearch=530574\&indexSearch=ID\#: :text=Resultado\%3A\%20o\%20Transv erso $\% 20$ do\%20abdomen,seu\%20trinamento\%20deve\%20ser\%20espec\%C3\%ADfico.

Hayden, J. A., Van Tulder, M. W., Malmivaara, A. V., \& Koes, B. W. (2005). Meta-analysis: exercise therapy for nonspecific low back pain. Ann Intern Med, 142(9), 765-75. https://doi.org/10.7326/0003-4819-142-9-200505030-00013

Hebert, J. J., Koppenhaver, S. L., Magel, J. S., \& Fritz, J. M. (2010). The relationship of transversus abdominis and lumbar multifidus activation and prognostic factors for clinical success with a stabilization exercise program: a cross-sectional study. Arch Phys Med Rehabil, 91(1), 78-85. https://doi.org/10.1016/j.apmr.2009.08.146

Hides, J., Gilmore, C., Stanton, W., \& Bohlscheid, E. (2008). Multifidus size and symmetry among chronic LBP and healthy asymptomatic subjects. Man Ther, 13(1), 43-49. https://doi.org/10.1016/j.math.2006.07.017

Hwangbo, G., Lee, C. W., Kim, S. G., \& Kim, H. S. (2015). The effects of trunk stability exercise and a combined exercise program on pain, flexibility, and static balance in chronic low back pain patients. J Phys Ther Sci, 27(4), 1153-5. https://doi.org/10.1589/jpts.27.1153 
Júnior, S., João, J., Michael K. N., Pimenta, C. A. M., Ali, A., \& Thieme, A. L. (2010). Validação do questionário de incapacidade de Roland Morris para dor em geral. Rev. dor, 11(1), 28-36. https://pesquisa.bvsalud.org/portal/resource/pt/lil-562427

Sensor Valedo [imagem online]. (2018). Hocoma. https://knowledge.hocoma.com/trainingmaterial/valedomotion/instruction-video.html.

Lizier, D. T., Perez, M. V., \& Sakata, R. K. (2012). Exercícios para tratamento de lombalgia inespecífica. Rev. Bras. Anestesiol, 62(6), 838-846. http://dx.doi.org/10.1590/S003470942012000600008 .

Macedo, L. G., Maher, C. G., Latimer, J., \& McAuley, J. H. (2009). Motor control exercise for persistent, nonspecific low back pain: a systematic review. Phys Ther, 89(1), 9-25. https://doi.org/10.2522/ptj.20080103.

Maia, F., Gurgel, F., Bezerra, J., \& Bezerra, C. (2015). Perspectivas terapêuticas da fisioterapia em relação à dor lombar. Revista da Faculdade de Ciências Médicas de Sorocaba, 17(4), 179-184. Recuperado de https://revistas.pucsp.br/index.php/RFCMS/article/view/18663.

Matheve, T., Bogaertes, K., \& Timmermans, A. (2020). Virtual reality distraction induces hypoalgesia in patients with chronic low back pain: a randomized controlled trial. J Neuroeng Rehabil, 22(1), 55. https://doi.org/10.1186/s12984-020-00688-0.

Nascimento, P. R. C., Costa, L. O. P. Prevalência da dor lombar no Brasil: uma revisão sistemática. Cad. Saúde Pública, 31(6), 1141-1156. http://dx.doi.org/10.1590/0102$311 X 00046114$.

Nieder, D., Engel, T., Vogt, L., Arampatzis, A., Banzer, W., Beck, H., Catalá, M. M., Brenner-Fliesser, M., Güthoff, C., Haag, T., Hönning, A., Pfeifer, A. C., Platen, P., Schiltenwolf, M., Schneider, C., Trompeter, K., Wippert, P. M., \& Mayer, F. (2020). Motor Control Stabilisation Exercise for Patients with Non-Specific Low Back Pain: A Prospective 
Meta-Analysis with Multilevel Meta-Regressions on Intervention Effects. J. Clin. Med, 9(9), 1-19. https://doi.org/10.3390/jcm9093058.

Niederer, D., \& Mueller, J. (2020). Sustainability effects of motor control stabilisation exercises on pain and function in chronic nonspecific low back pain patients: a systematic review with meta-analysis and meta-regression. Plos One, 15(1), 1-21.

https://doi.org/10.1371/journal.pone.0227423.

Patiño,A.G., Khoshnam, M., Menon, C. (2020) Wearable Device to Monitor Back Movements Using an Inductive Textile Sensor. Sensors, 20, 905; https://doi:10.3390/s20030905.

Pereira A.S., Shitsuka D.M., Parreira, F.J., Shitsuka. R. (2018). Metodologia da pesquisa científica. [e-book]. Santa Maria. Ed. UAB/NTE/UFSM. Disponível em: https://repositorio.ufsm.br/bitstream/handle/1/15824/Lic_Computacao_MetodologiaPesquisa-Cientifica.pdf? sequence $=1$.

Santos, R. M., Freitas, D. G., Pinheiro, I. C. O., Vantin, K., Gualberto, H. D., \& Carvalho, N. A. A. (2011). Estabilização Segmentar Lombar. Med. reabil, 30(1), 14-17. https://pesquisa.bvsalud.org/portal/resource/pt/lil-597964.

Shahvarpour, A., Gagnon, D., Preuss, R., Henry, S. M., \& Larivière, C. (2018). Trunk postural balance and low back pain: Reliability and relationship with clinical changes following a lumbar stabilization exercise program. Gait Posture, 61(1), 375-381. https://doi.org/10.1016/j.gaitpost.2018.02.006.

Sipaviciente, S., \& Kliziene, I. (2020). Effect of different exercise programs on non specific chronic low back pain and disability in people who perform sedentary work. Clin Biomech, 73(1), 17-27. https://doi.org/10.1016/j.clinbiomech.2019.12.028.

Soundararajan, L. R. A., \& Thankappan, S. M. (2016). Efficacy of the Multifidus Retraining Program in Computer Professionals with Chronic Low Back Pain. Asian Spine J, 10(3), 450456. https://doi.org/10.4184/asj.2016.10.3.450. 
Sowden, G., Hill, J. C., Morso, L, Louw, Q, \& Foster, NEF. (2018) Advancing practive for back pain through stratified care (STarT Back). Brazilian Journal of Physical Therapy, 22(4), 255-264. 10.1016/j.bjpt.2018.06.003.

Stollenwerk, K., Müller, J., Hinkenjann, A., \& Krüger, B. (2019). Analyzing Spinal Shape Changes During Posture Training Using a Wearable Device. Sensors, 19(16), 1-21. https://doi.org/10.3390/s19163625.

\section{Porcentagem de contribuição de cada autor no manuscrito}

Rafael Victor Ferreira do Bonfim - 20\%

Thayanne Kelly de Sousa Cabral - 20\%

Rayane Portela de Lima - 20\%

Bruno da Silva Gomes - 10\%

Gabriel Mauriz de Moura Rocha - 10\%

$$
\text { Livia Assis - 10\% }
$$

Vilson Rosa Almeida - 10\% 